\title{
Examinando o fenômeno da corrupção no mundo: Um olhar sobre os fatores que interferem na percepção da corrupção
}

\author{
Examining the phenomenon of corruption around the world: A look over the factor that interferer \\ in corruption perception \\ Examinando el fenómeno de la corrupción en el mundo: Una mirada a los factores que interfieren \\ en la percepción de la corrupción
}

\author{
Eliã Pereira de Oliveira \\ ORCID: https://orcid.org/0000-0002-2884-6513 \\ Universidade Federal Fluminense, Brasil \\ E-mail: eliaoliveira@id.uff.br \\ João Alberto Neves dos Santos \\ ORCID: https://orcid.org/0000-0002-4812-6214 \\ Universidade Federal Fluminense, Brasil \\ E-mail: joaoneves@id.uff.br \\ José Paulo Cosenza \\ ORCID: https://orcid.org/0000-0002-1753-0134 \\ Universidade Federal Fluminense, Brasil \\ E-mail: jpcosenza@id.uff.br \\ Samantha Fernandes Milczanowski Neves \\ ORCID: https://orcid.org/0000-0002-6406-9707 \\ Universidade Federal do Rio de Janeiro, Brasil \\ E-mail: samantha.milczanowski@uerj.br
}

\begin{abstract}
Resumo
O objetivo do artigo é identificar quais variáveis podem influenciar nos Índices de Percepção da Corrupção dos países, por meio de uma análise da atuação simultânea de variáveis relacionadas aos fatores social, político, jurídico e econômico. A metodologia da pesquisa compreende então a construção de um modelo de regressão linear múltipla, representativo da relação entre os Índices de Percepção da Corrupção dos países e conjunto de variáveis analisadas. Para a coleta de dados, informações disponibilizadas por vários sítios de internet internacionais (Transparency International, NationMaster database, World Bank, Fraser Institute of Economics, Freedom House and the United Nations) foram acessadas, a partir das quais criou-se um banco de dados para a realização da análise de regressão linear múltipla, seguindo os pressupostos desta técnica e, considerando uma modelagem com quatro hipóteses. Os resultados encontrados apontaram que, ao serem analisadas simultaneamente, as variáveis estaticamente significantes do modelo (p-value<=10) estão relacionadas aos fatores: i) político (Liberdade Civil e Direitos Políticos), ii) social (Educação e Renda Nacional Bruta Per Capita) e iii) jurídico (Imparcialidade do Júri e Integridade do Sistema Jurídico). Quanto as variáveis relacionadas ao fator econômico, não foram encontradas evidências estatísticas (pvalue>0,10) de que as mesmas influenciem nos Índices de Percepção da Corrupção. A pesquisa aqui abordada auxilia os formuladores de políticas públicas dos países na concepção de políticas públicas que visem influenciar, de forma positiva, no Índice de Percepção da Corrupção destes.
\end{abstract}

Palavras-chave: Corrupção; Governança; Causas da corrupção; Proteção à corrupção.

\begin{abstract}
The objective of the article is to identify which variables can influence the Corruption Perception Indexes of the countries, through an analysis of the simultaneous action of variables related to social, political, legal and economic factors. The research methodology then comprises the construction of a multiple linear regression model, representative of the relationship between the Corruption Perception Indices of the countries and the set of analyzed variables. For data collection, information provided by several international internet sites (Transparency International, NationMaster database, World Bank, Fraser Institute of Economics, Freedom House and the United Nations) was accessed, from which a database was created. To perform the multiple linear regression analysis, following the assumptions of this technique and considering a model with four hypotheses. The results found showed that, when analyzed simultaneously, the statistically significant variables of the model ( $p$-value $<=10$ ) are related to factors: i) political (Civil Freedom and Political Rights), ii) social (Education and Gross National Income Per Capita) and iii) legal (Impartiality of the Jury and Integrity of the Legal System). As for the variables related to the economic factor,
\end{abstract}


no statistical evidence was found (p-value>0.10) that they influence the Corruption Perception Indices. The research addressed here helps public policy makers in countries to design public policies that aim to positively influence their Corruption Perception Index.

Keywords: Corruption; Good governance; Causes of corruption; Protection against corruption.

\section{Resumen}

El objetivo del artículo es identificar qué variables pueden influir en los Índices de Percepción de la Corrupción de los países, mediante un análisis de la acción simultánea de variables relacionadas con factores sociales, políticos, legales y económicos. Luego, la metodología de investigación comprende la construcción de un modelo de regresión lineal múltiple, representativo de la relación entre los Índices de Percepción de la Corrupción de los países y el conjunto de variables analizadas. Para la recopilación de datos se accedió la información proporcionada por varios sitios internacionales de Internet (Transparency International, NationMaster database, World Bank, Fraser Institute of Economics, Freedom House and the United Nations), a partir de la cual se creó una base de datos para realizar el análisis de regresión lineal múltiple, siguiendo los supuestos de esta técnica y considerando un modelo con cuatro hipótesis. Los resultados encontrados mostraron que, cuando se analizan simultáneamente, las variables estadísticamente significativas del modelo ( $\mathrm{p}$-value $<=10$ ) se relacionan con factores: i) políticos (Libertad Civil y Derechos Políticos), ii) sociales (Educación e Ingreso Nacional Bruto) Per cápita) y iii) legal (Imparcialidad del Jurado e Integridad del Sistema Legal). En cuanto a las variables relacionadas con factor económico, no se encontró evidencia estadística suficiente de que influyan en los Índices de Percepción de la Corrupción. La investigación que se analiza aquí ayuda a los formuladores de políticas públicas de los países en el diseño de políticas públicas que tienen como objetivo influir positivamente en su Índice de Percepción de la Corrupción.

Palabras clave: Corrupción; Gobernancia; Causas de la corrupción; Protección contra la corrupción.

\section{Introdução}

A corrupção pode ser estudada tanto no nível macro (país), micro (indivíduo) como em ambos conjugadamente (O’Connor e Fischer, 2012). Trata-se, portanto, de um problema comportamental, cujos reflexos impactam diversos segmentos da sociedade em todo o mundo (Brol, 2016). Apesar dos prejuízos causados pelos escândalos de corrupção em todos os níveis, trata-se de uma agenda que ainda tem sido abordada marginalmente em eventos acadêmicos e científicos (Medeiros e Silveira, 2017) e apenas mais recentemente vem recebendo uma maior atenção por parte da academia (Oberoi, 2014) e obtendo maiores espaços em publicações de periódicos científicos (Ashforth, Gioia, Rovinson, \& Treviño, 2008; Zyglidopoulos, Hirsch \& Holan, 2017; Journal of Management Inquiry, 2017; Revista de Contabilidade e Organizações, 2019).

Ao longo da história da humanidade é significativa a presença da corrupção em diferentes momentos, sociedades, regimes e culturas (Alatas, 1990), suas práticas afetam os sistemas econômicos, políticos e legais dos países, podendo também alterar o desenvolvimento de uma comunidade e impedir um nível maior de crescimento social e melhoria de qualidade de vida da população (Luciano, Wiedenhöft, \& Callegaro, 2019).

Dentre os diversos setores da economia de um país, o mais propenso a casos de corrupção é o da construção civil, sendo apontado por diversas vezes pelo Transparency International (2019) em seus relatórios "Bribe Payers Index" como o segmento mais provável de ocorrer pagamentos de suborno. A American Society of Civil Engineers, por sua vez, demonstra que a corrupção na indústria da construção civil consome um valor estimado da ordem de US\$ 340 bilhões, por ano, em todo o mundo (Asce, 2004).

Por se tratar de um setor socioeconomicamente importante para um país (Ive e Gruneberg, 2000; Anaman \& Amponsah, 2007; Stansbury, 2005; Unctad, 2000), é necessário que sejam realizadas análises, de modo a identificar os fatores que mais influenciam a corrupção e sua relação com a indústria da construção civil. Recentemente, foram desenvolvidas pesquisas com o intuito de levantar as formas de corrupção nesse setor, buscando compreender suas causas e os comportamentos que levam a tal prática nesse segmento econômico (Nordin, Takim, \& Nawawi, 2013; Chan \& Owusu, 2017; Valarini \& Pohlmann, 2019).

Além do fator econômico que pode fortemente influenciar a corrupção, outras abordagens estão sendo estudadas para 
verificar como o combate à corrupção está sendo vitorioso, baseando-se no apoio a diversos fatores. No que tange ao fator jurídico, Goel e Nelson (2010) identificaram que, os países com sistema jurídico do tipo "direito comum" (common law), caso de países de origem anglo-saxônica como Estados Unidos, Inglaterra, Canadá, dentre outros, tendem a possuir baixos níveis de corrução.

Em relação ao fator social, Alves, Curta e Dias (2017) afirmam que países com os menores níveis de corrupção tendem a possuir maior desenvolvimento humano (educação, saúde e renda per capita). Kolstad e Wiig (2016), por sua vez, partindo de um ponto de vista político, verificaram o efeito positivo da democracia para a redução da corrupção.

Diante disso, foi identificada uma oportunidade de avançar no conhecimento que se tem sobre este tema, construindo um modelo de regressão múltipla com dados encontrados sobre os fatores econômicos, sociais, jurídicos e políticos e os índices de corrupção dos países. Por conseguinte, esta pesquisa teve por objetivo identificar quais variáveis, dentro dos fatores analisados (sociais, políticos, jurídicos e econômicos), possuem maior poder de influência na variação dos níveis de 'corrupção' dos países, quando examinadas de forma simultânea.

Esta pesquisa se justifica por meio de três aspectos: (i) a corrupção tem sido um tema atual e de interesse global pelas empresas, uma vez que têm buscado estarem em conformidade com as leis, regras e regulamentos ("compliance"), para assim se destacarem no mercado em que atuam; (ii) a construção civil é afetada severamente pela corrupção, em si, uma vez que traz uma série de consequências, dentre elas o aumento do custo total do empreendimento, a redução da qualidade da infraestrutura e os atrasos; e (iii) além de impactar negativamente, a construção civil também afeta toda a economia de um país, visto que esta indústria possui uma parcela relevante do PIB.

Face ao exposto, adota-se uma perspectiva interdisciplinar, cuja essência concentra-se na discussão de uma problemática prática que se circunscreve na intersecção entre as áreas de Engenharia Civil, Arquitetura e Urbanismo, Administração, Contabilidade, Economia e Ciências Políticas. Os resultados desta análise auxiliarão, portanto, na provisão do conhecimento sobre um tema de vital importância à economia, a política e a sociedade, dentre outros, uma vez que a corrupção envolve uma série de riscos nocivos ao país.

\subsection{Definição da corrupção e seus fatores relacionados}

A Transparency International (2018), corroborando com Nye (1967), define corrupção como "o abuso do poder confiado, visando obter benefícios privados", pois ela pode acontecer em qualquer lugar e envolver todo tipo de pessoa, podendo ser classificada em grandes ou pequenas, de acordo com a quantia desviada, e em sistêmica ou política, conforme o âmbito, setor ou estrato social em que ocorre. Boyd e Padilla (2009), de forma mais específica, expõe que a corrupção é composta de três componentes fundamentais: (i) a demanda que apela por corrupção; (ii) a oferta que proporciona a mesma; e (iii) a parte condescendente que tem ciência de quando a referida ocorre, porém permanece em silêncio ou indiferente diante da situação.

Ao abordar demandas emergentes relativas à corrupção e repensar velhas questões à luz de novos dados, expandindo o tratamento da cultura como fonte de corrupção enraizada, Rose-Ackerman e Palifka (2016) apresentam um esquema gráfico, que ilustra as causas e consequências da corrupção, conforme exposto na Figura 1. Tal esquema é oriundo das descobertas mais robustas de uma série de pesquisas empíricas, tanto em esfera macro, entre países, como também micro, utilizando-se dados mais específicos sobre a corrupção.

Rose-Ackerman e Palifka (2016) salientam que as setas apontando para uma única direção foram utilizadas apenas para fins de simplicidade de entendimento, pois em diversos casos a mesma pode apontar para ambos os lados, isto é, como causa e consequência da corrupção ao mesmo tempo. Por exemplo, no caso de um estado de direito deficiente, este contribui para corrupção do mesmo modo que a corrupção impacta negativamente nesse. 
Figura 1. Causas e consequências da corrupção.

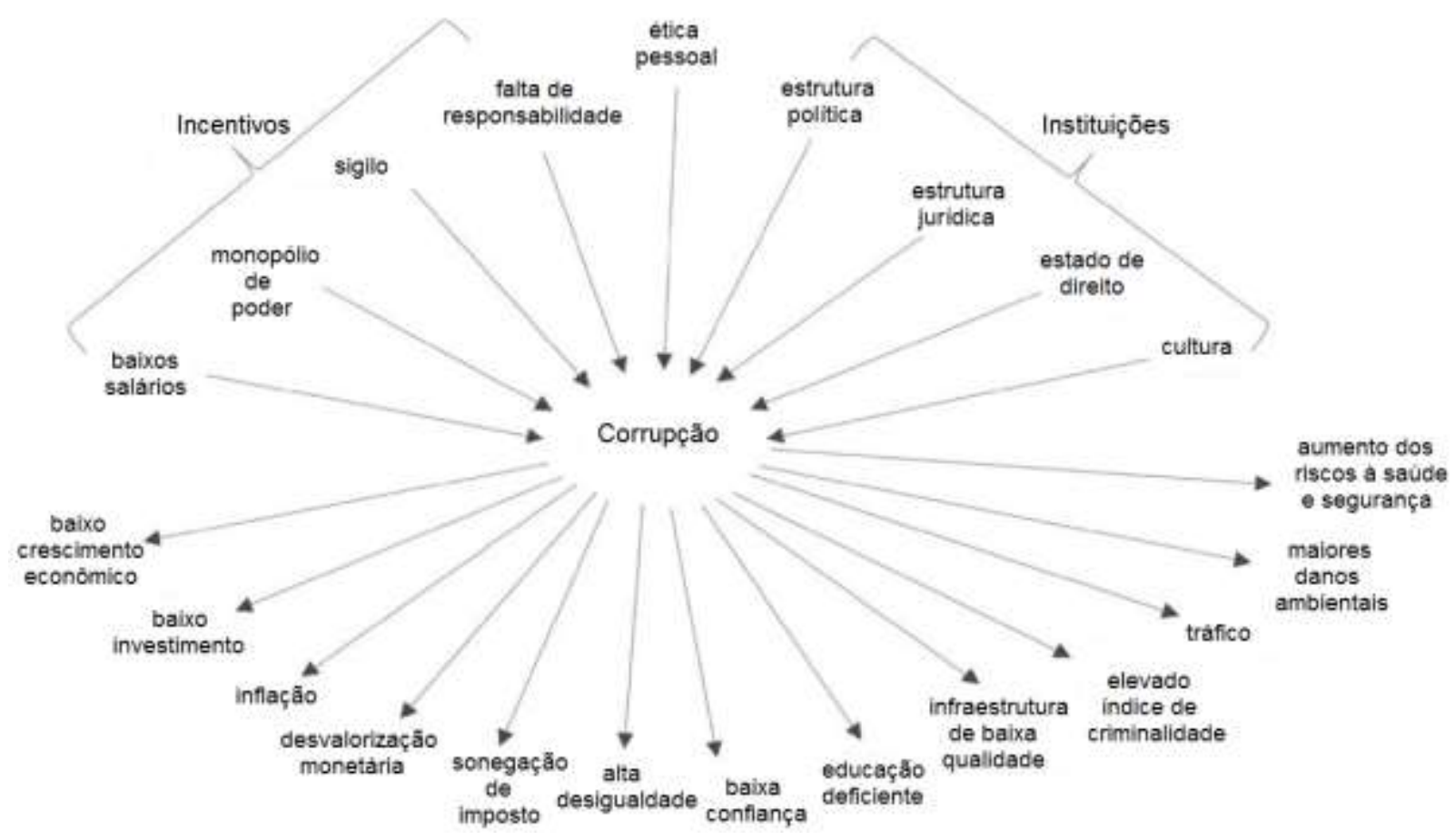

Fonte: Extraído de Rose-Ackerman e Palifka (2016).

Dentre os diversos fatores influenciadores da corrupção está o econômico. Estudos têm sido realizados com o intuito de compreender melhor o comportamento entre as variáveis desse fator e a corrupção, buscando, assim, contribuir para o combate da corrupção. Lučić, Radišić e Dobromirov (2016) e Cieślik e Goczek (2018) constataram a existência de uma relação direta entre o crescimento das variáveis PIB e PIB Per Capita de um país e a redução da corrupção no mesmo.

No que se referem à construção civil, pesquisas concluem que a corrupção neste setor é mais intensa que em qualquer outro setor da economia, sendo identificadas práticas de corrupção em todas as fases de construção de um empreendimento, isto é, planejamento e projeto, adjudicação de contratos, construção e, por fim, operação e manutenção (Transparency International, 2002; Stansbury, 2005).

Além disso, outra variável do fator econômico que também possui influência na corrupção é a inflação. Akça, Ata e Karaca (2012) identificaram que ela impacta a integridade de país de modo a intensificar a corrupção na sociedade.

Em se tratando do Fator Social, uma das variáveis mais importantes para os países é o índice de desenvolvimento humano, popularmente conhecido com IDH, que se baseia nos indicadores sociais de renda, educação e saúde. No que se referem à renda, alguns autores verificaram que a igualdade de renda ou a renda per capita elevada contribuem para a redução da corrupção na sociedade (Chong \& Gradstein, 2007; Apergis, Dincer \& Payne, 2010; Mendonça e Fonseca, 2012), por outro lado, Dobson e Ramlogan-Dobson (2010) observaram que, para o caso da América Latina, quanto maior renda per capita da população, maior tende a ser a corrupção.

Com relação à variável educação, diversos autores constataram que investimentos em educação favorecem a sociedade, combatendo a corrupção através da instrução e estímulo a práticas íntegras (Sofwan et al., 2018; Ferraz, Finan \& Moreira, 2012; Asongu \& Nwachukwu, 2015), porém a baixa escolaridade é uma deficiência a ser enfrentada (Valinote et al.; 2014).

A questão jurídica representa outro fator que tem sido objeto de estudo para se entender melhor o comportamento da corrupção nos países. No que diz respeito à variável integridade do sistema jurídico, em suas pesquisas, Uslaner (2008) e 
Montes e Luna (2020) identificaram que países com baixos níveis de corrupção tendem a possuir também sistemas jurídicos mais íntegros e justos.

No que diz respeito à imparcialidade jurídica, Bennedsen, Feldmann e Lassen (2011) constataram que esta, de modo geral, correlaciona-se negativamente com a corrupção, atuando como forte preditor de práticas de suborno.

Sobre a variável independência judicial, Cordis (2009) e Mungiu-Pippidi e Dadašov (2016) reconheceram, através de suas pesquisas, que esta tende a ser uma das ferramentas eficazes no controle da corrupção da sociedade.

Em relação ao estado de direito (rule of law), Elbahnasawy e Revier (2012) concluíram que este sistema institucional tem forte impacto no combate à corrupção, sendo até mesmo mais importante que outras variáveis, como, por exemplo, a Renda Per Capita.

No que concerne ao Fator Político, tem sido considerada a variável democracia, baseando-se nas questões da liberdade civil e direitos políticos. Pellegrini (2011) e Saha et al. (2014) verificaram que a democracia contribui positivamente para uma sociedade menos corrupta, sendo crucial para o controle da corrupção. Contudo, Jetter, Agudelo e Hassan (2015) mostram que a liberdade civil e direitos políticos só favorecem o combate à corrupção em países de renda per capita mais elevada. Nos países mais pobres esta tende a contribuir para o aumento da corrupção.

\subsection{Hipóteses Formuladas}

Com base em toda a literatura de referência apresentada, foram estabelecidas as seguintes hipóteses de pesquisa:

Hipótese 1: As variáveis de fator econômico (Crescimento do PIB, PIB da Construção Civil e Inflação) estão diretamente relacionadas à percepção da corrupção.

Hipótese 2: As variáveis de fator social (Renda Bruta Per Capita e Índices de Educação) estão inversamente relacionadas à percepção da corrupção.

Hipótese 3: As variáveis de fator jurídico (Integridade do Sistema Jurídico, Imparcialidade do Júri, Independência Judicial e Estado de Direito) estão inversamente relacionadas à percepção da corrupção.

Hipótese 4: A variável de fator político (Liberdade Civil e Direitos Políticos) está inversamente relacionada à percepção da corrupção.

\section{Metodologia}

Com base na exposição feita sobre o assunto abordado e diante do objetivo referido, apresenta-se o tipo de pesquisa realizada como bibliográfica, de caráter exploratório e com abordagem quantitativa.

A pesquisa bibliográfica trata-se de uma forma de discutir e explicar um determinado tema ou problema, baseando-se em referências publicadas, visando assim contribuir para com o conhecimento teórico e científico do estudo em questão (Martins \& Theóphilo, 2016); quanto ao caráter exploratório, Gil (2008) esclarece que este é o que tem como fim desenvolver ou elucidar conceitos sobre um tema não muito explorado; e uma abordagem quantitativa refere-se àquela realizada por meio de ferramentas estatísticas para medir as relações existentes entre as variáveis analisadas, gerando assim um resultado final quantificável (Fonseca, 2002).

Diante do que foi apresentado por Rose-Ackerman e Palifka (2016), descrito na Figura 1 e com base nos fatores de influência da corrupção conforme expostos anteriormente, buscou-se por meio da construção de um modelo de regressão linear múltipla, verificar, de forma empírica e estatística, quais variáveis dos fatores econômico, social, jurídico e político, quando analisadas de forma conjunta e simultânea, verdadeiramente atuam influenciando nos índices de percepção da corrupção dos países. 
A regressão linear múltipla é uma ferramenta estatística eficaz na identificação de variáveis que possuem relação de causa e efeito entre si (Uyanik \& Guler, 2013). Seu processo possui como resultado uma equação que representa o comportamento linear da relação entre a variável dependente e as independentes (Hair et al., 2019; Tabachnick \& Fidell, 2013).

Para a construção do modelo foram utilizados os valores das variáveis encontradas nos fatores citados e os índices de percepção da corrupção de cada país. A pesquisa considerou então, uma amostra, estruturada no formato de corte transversal (cross-section), delimitada a 125 países, dos 180 listados pela Transparência Internacional em seu Índice de Percepção da Corrupção (IPC) de 2017. A exclusão de 55 países da amostra deu-se por não apresentarem dados referentes a uma ou mais variáveis dos fatores considerados neste estudo.

Ressalta-se, também, que se definiu o ano de 2017 por ser o ano mais atual dos registros das variáveis encontradas para os fatores jurídicos, servindo como ano de referência para a obtenção de todas as demais variáveis de interesse.

A Tabela 1 apresenta resumidamente todas as variáveis analisadas na pesquisa, suas fontes de origem e fatores aos quais estão relacionadas.

Tabela 1. Resumo dos dados.

\begin{tabular}{llc}
\hline \multicolumn{1}{c}{ Fatores } & \multicolumn{1}{c}{ Variáveis Analisadas } & Fonte \\
\hline Corrupção & Índices de Percepção da Corrupção & Transparency International \\
\hline \multirow{3}{*}{ Econômicos } & PIB da Construção Civil (\%) & NationMaster \\
& Crescimento do PIB (\%) & WorldBank \\
& Inflação (\%) & Fraser Institute \\
\hline \multirow{3}{*}{ Jurídicos } & Independência Judicial & Fraser Institute \\
& Imparcialidade do Júri & Fraser Institute \\
& Integridade do Sistema Jurídico & Fraser Institute \\
Políticos & Estado de Direito (Rule of Law) & WorldBank \\
\hline \multirow{2}{*}{ Sociais } & Liberdade civil e Direitos Políticos (Democracia) & Freedom House \\
& Renda Nacional Bruta Per Capita & WorldBank \\
\hline
\end{tabular}

Fonte: Autores (2021).

É importante mencionar que os dados obtidos foram norteados pelas variáveis de influência da corrupção expostas na Figura 1. Entretanto, em virtude da dificuldade de obtenção de dados, algumas variáveis apresentadas nesta figura não puderam ser incorporadas na construção do modelo de regressão linear múltipla proposto. Com exceção de "Índices de Percepção da Corrupção", que se trata da variável dependente do modelo, todas as demais variáveis expostas na Tabela 1 foram consideradas como independentes.

Por se tratar de uma análise de regressão linear múltipla, Williams, Grajales e Kurkiewicz (2013) esclarecem que é necessário obedecer aos seguintes pressupostos: (i) identificação e correção dos erros observacionais; (ii) verificação de multicolinearidade entre as variáveis preditoras; (iii) variância constante dos erros (homocedasticidade); (v) verificação de outliers; (vi) linearidade nos parâmetros da regressão múltipla; (vii) média condicional zero dos erros; (viii) distribuição normal dos erros; (xix) independência dos erros.

Sobre o pressuposto de identificação e correção dos erros observacionais, este foi assumido em virtude de os dados obtidos já terem sido tratados, de forma apropriada, pelos devidos órgãos de origem. Para a verificação do processo 
metodológico envolvido na construção dos dados de cada uma das variáveis analisadas, consultar as fontes apresentadas na Tabela 1.

Na verificação dos demais pressupostos, utilizou-se o software SPSS 21.0. Os resultados estatísticos apresentados por este, informam o valor p ou p-value, isto é, da significância estatística dos testes executados. Logo, definiu-se a regra de decisão dos testes estatísticos, conforme indicado por Boslaugh e Watters (2008), em:

- p-value $\leq$ 0,05: Rejeição da hipótese nula (H0);

- p-value > 0,05: Não rejeição da hipótese nula (H0).

Definida a regra de decisão, iniciou-se a busca da identificação do modelo de regressão final, aceitando somente as variáveis significantes, estatisticamente, do modelo. Para isso, verificou-se, primeiro, a presença de variáveis colineares entre si, através do Variance Inflation Factor (VIF) de cada variável. Os resultados de VIF apresentados pelo SPSS mostraram a necessidade de que as variáveis Estado de Direito e Independência Judicial fossem retiradas do modelo final, dado que seus valores foram 19,162 e 6,273, respectivamente, valores esses superiores a 5, conforme indicado para remoção por Akinwande, Dikko e Samson (2015).

Retiradas as variáveis colineares, passou-se, então, à verificação de quais variáveis poderiam permanecer no modelo final, tendo em vista a adequação dos coeficientes das variáveis preditoras. Para isso, foram analisados, a cada rodada de verificação, a significância estatística (p-value) de cada variável. Os valores encontrados são apresentados na Tabela 2 a seguir:

Tabela 2. Significância estatística das variáveis preditoras.

\begin{tabular}{lc}
\hline \multicolumn{1}{c}{ Variável } & p-value \\
\hline PIB da Construção Civil (\%) & 0,679 \\
Inflação (\%) & 0,090 \\
Crescimento PIB (\%) & 0,384 \\
\hline
\end{tabular}

Fonte: Autores (2021).

As variáveis inconsistentes foram removidas do modelo de regressão, restando somente três variáveis com significância estatística no comportamento da variável Índice de Transparência: PIB da Construção Civil (R\$), Índice de Educação e Rendimento Per Capita (R\$).

As referidas variáveis foram então retiradas do modelo, restando assim, cinco variáveis estatisticamente significantes na variação dos Índices de Percepção da Corrupção: Liberdade Civil e Direitos Políticos (Democracia), Índices de Educação, Imparcialidade do Júri, Integridade do Sistema Jurídico e Renda Nacional Bruta Per Capita. Sendo estas, assim, validadas como estatisticamente significantes no comportamento da variável "Índices de Corrupção".

Dando prosseguimento à verificação dos pressupostos, realizou-se no SPSS o teste de homocedasticidade de BreuschPagan. Inicialmente, o resultado calculado indicava que o modelo final era heterocedástico, com p-value igual a 0,008, não obedecendo ao pressuposto de homocedasticidade ( $\mathrm{p}$-value $>=0,05$ ). A partir daí, buscou-se identificar a presença de outliers multivariados no modelo de regressão, por meio da distância de Mahalanobis (1936).

Calculou-se, portanto, esta distância para cada país analisado e em concordância com as variáveis do modelo final. Após a transformação dos diversos valores de distância de Mahalanobis em p-values, uma lista de p-values referentes aos valores desta distância foi então criada. Baseando-se então em Kline (2010), para p-values da distância de Mahalanobis menores que 0,001, os respectivos dados seriam considerados outliers multivariados. De início, dois países se apresentaram como outliers do modelo, Brunei e Qatar, e em uma segunda verificação também foi removido o Kuwait. Após a remoção dos dados desses três países, refez-se o teste de homocedasticidade que apresentou o resultado de 0,098, sendo assim constatado como homocedástico. 
Para a verificar a linearidade dos parâmetros da regressão, foi avaliado o p-value da tabela da ANOVA, em que a hipótese nula (H0) estabelece a inexistência de linearidade significativa entre as variáveis independentes e dependente. $\mathrm{O}$ valor de p-value identificado foi de 0,000, consistindo assim, na rejeição da hipótese nula, isto é, o modelo de regressão final segue uma linearidade significativa.

Na verificação da média condicional dos erros igual a zero, foram gerados os erros não padronizados, a partir do modelo de regressão final. Com base na distribuição dos erros, calculou-se a média e obteve-se um valor igual a zero, atendendo assim a esse pressuposto.

$\mathrm{Na}$ verificação da média condicional dos erros igual a zero, a partir do modelo de regressão final, foram gerados os valores dos erros não padronizados. De posse, da distribuição dos erros, calculou-se então a média, que por sua vez apresentou valor zero, atendendo então este pressuposto.

Já na verificação da normalidade dos erros, realizou-se o teste de Kolmogorov-Smirnov nos erros anteriormente obtidos, por se tratar de uma amostra grande. O resultado apresentado foi de 0,200 , que se traduz na não rejeição da hipótese nula, isto é, os erros seguem uma distribuição normal.

Finalmente, para verificação do último pressuposto (independência dos erros), realizou-se o teste de Durbin-Watson, gerando como resultado o valor 2,036. Extrapolou-se então este valor, para obtenção da significância estatística, por meio da tabela de Durbin-Watson, considerando tamanho da amostra igual a 122 e 5 regressores. Verificou-se então que dL=1,665 e $\mathrm{dU}=1,802$, o que implica em mostra que 4-dU=2,198 e 4-dL=2,335. Logo, o valor calculado está na faixa de 1,802 e 2,198, levando assim a constatação de que os erros do modelo são independentes.

\section{Resultados e Discussão}

Ao final de todo o processo metodológico, constatou-se que o modelo de regressão que melhor descreve a variação dos Índices de Percepção da Corrupção dos países é composto pelas variáveis Imparcialidade do Júri, Integridade do Sistema Jurídico, Liberdade Civil e Direitos Políticos, Renda Nacional Bruta Per Capita e Índices de Educação. Essa afirmação é comprovada pelo poder explicativo do modelo $\left(\mathrm{R}^{2}\right)$, cujo valor é de 0,907 .

A existência da relação entre as variáveis do modelo é comprovada pelos valores dos coeficientes de correlação de Pearson apresentados na Tabela 3 deste artigo. Além disso, a Tabela 4, expõe os valores das estatísticas descritivas das variáveis do modelo de regressão.

Tabela 3. Coeficientes de Correlação de Pearson.

\begin{tabular}{lcccccc}
\hline & $\begin{array}{c}\text { Índice de Percepção } \\
\text { da Corrupção }\end{array}$ & $\begin{array}{c}\text { Renda } \\
\text { Per } \\
\text { Capita }\end{array}$ & $\begin{array}{c}\text { Liberdade } \\
\text { Civil e } \\
\text { Política }\end{array}$ & $\begin{array}{c}\text { Índice de } \\
\text { Educação }\end{array}$ & $\begin{array}{c}\text { Imparcialidade do } \\
\text { Júri }\end{array}$ & $\begin{array}{c}\text { Integridade do } \\
\text { Sistema } \\
\text { Jurídico }\end{array}$ \\
\hline $\begin{array}{l}\text { Índice de } \\
\text { Percepção da } \\
\text { Corrupção }\end{array}$ & 1 & 0,867 & 0,602 & 0,733 & 0,708 & 0,775 \\
\hline $\begin{array}{l}\text { Renda Per } \\
\text { Capita }\end{array}$ & 0,867 & 1 & 0,373 & 0,788 & 0,617 & 0,726 \\
\hline $\begin{array}{l}\text { Liberdade Civil } \\
\text { e Política }\end{array}$ & 0,602 & 0,373 & 1 & 0,462 & 0,204 & 0,394 \\
\hline $\begin{array}{l}\text { Índice de } \\
\text { Educação }\end{array}$ & 0,733 & 0,788 & 0,462 & 1 & 0,340 & 0,594 \\
\hline $\begin{array}{l}\text { Imparcialidade } \\
\text { do Júri }\end{array}$ & 0,708 & 0,617 & 0,204 & 0,340 & 1 & 0,607 \\
\hline $\begin{array}{l}\text { Integridade do } \\
\text { Sistema } \\
\text { Jurídico }\end{array}$ & 0,775 & 0,726 & 0,394 & 0,594 & 0,607 & 1 \\
\hline
\end{tabular}


Fonte: Autores (2021).

Tabela 4. Estatística Descritiva.

\begin{tabular}{lccccc}
\hline Variáveis & $\mathbf{N}$ & Mínimo & Máximo & Média & Desvio Padrão \\
\hline $\begin{array}{l}\text { Índice de } \\
\text { Percepção da } \\
\text { Corrupção }\end{array}$ & 122 & 17 & 89 & 46,16 & 19,386 \\
\hline $\begin{array}{l}\text { Renda Per } \\
\text { Capita }\end{array}$ & 122 & 0,086 & 8,984 & 2,246 & 1,997 \\
\hline $\begin{array}{l}\text { Liberdade Civil } \\
\text { e Política }\end{array}$ & 122 & 1 & 3 & 2,330 & 0,732 \\
\hline $\begin{array}{l}\text { Índice de } \\
\text { Educação }\end{array}$ & 122 & 0,245 & 0,946 & 0,685 & 0,175 \\
\hline $\begin{array}{l}\text { Imparcialidade } \\
\text { do Júri }\end{array}$ & 122 & 0,619 & 7,965 & 4,292 & 1,563 \\
\hline $\begin{array}{l}\text { Integridade do } \\
\text { Sistema Jurídico }\end{array}$ & 122 & 1,667 & 10 & 6,052 & 2,161 \\
\hline
\end{tabular}

Fonte: Autores (2021)

As variáveis que então compõem o modelo de regressão são apresentadas na Tabela 5 a seguir:

Tabela 5. Coeficientes da regressão linear múltipla.

\begin{tabular}{lccc}
\hline \multicolumn{1}{c}{ Variáveis do Modelo } & $\begin{array}{c}\text { Coeficientes não } \\
\text { Padronizados }\end{array}$ & $\begin{array}{c}\text { Coeficientes } \\
\text { Padronizados }\end{array}$ & $\begin{array}{c}\text { Significância } \\
\text { Estatística }\end{array}$ \\
\hline $\begin{array}{l}\text { Índice de Percepção da } \\
\text { Corrupção (constante) }\end{array}$ & $-10,841$ & & 0,005 \\
\hline $\begin{array}{l}\text { Liberdade Civil e } \\
\text { Direitos Políticos }\end{array}$ & 7,777 & 0,294 & 0,000 \\
\hline Índices de Educação & 11,523 & 0,104 & 0,043 \\
\hline Imparcialidade do Júri & 3,512 & 0,283 & 0,000 \\
\hline $\begin{array}{l}\text { Integridade do Sistema } \\
\text { Jurídico }\end{array}$ & 1,170 & 0,130 & 0,004 \\
\hline $\begin{array}{l}\text { Renda Nacional Bruta } \\
\text { Per Capita }\end{array}$ & 3,943 & 0,406 & 0,000 \\
\hline
\end{tabular}

Fonte: Autores (2021)

Da Tabela 5 nota-se que cinco variáveis foram descartadas do modelo sendo estas PIB da Construção Civil (\%), Crescimento do PIB (\%), Inflação (\%), Independência Judicial e Estado de Direito. Estas foram descartadas por questões de multicolinearidade e significância estatística não relevante. Entretanto, apesar das referidas terem sido descartadas, não significa que estas não possuam relação com a percepção da corrupção, e sim, que não foram encontradas evidências estatísticas suficientes para mantê-las no modelo de regressão.

Os resultados encontrados sugerem a rejeição da Hipótese 1 (as variáveis de fator econômico estão diretamente relacionadas à percepção da corrupção) uma vez que as variáveis ao serem analisadas simultaneamente com as variáveis dos 
demais fatores, não são estatisticamente significantes no aumento ou redução da corrupção, discordando assim das inferências de Lučić, Radišić e Dobromirov (2016) e Cieślik e Goczek (2018), de que a corrupção reduz a taxa de crescimento do PIB de um país, ou mesmo os resultados de Akça, Ata e Karaca (2012), de que a inflação tende a contribuir para com o aumento da corrupção em um país.

Ainda sobre a Hipótese 1, também foi constatado que, apesar da Construção Civil ser um setor da economia de extrema propensão a apresentar casos de corrupção, devido a uma série de aspectos, tais como complexidade, envolvimento do governo, número de ligações contratuais entre empresas, entre outros (Transparency International, 2018; Stansbury, 2005), não foram encontradas evidências significativas na pesquisa de que um mercado de Construção Civil aquecido, traduzido por um elevado PIB da Construção Civil, influencie na corrupção.

Estes resultados e a consequente sugestão de rejeitar a Hipótese 1 podem ser explicados pelo fato de que, a partir dos dados que foram utilizados nesta pesquisa, não houve evidências estatísticas suficientes para considerar as variáveis do Fator Econômico como influentes na percepção da corrupção, quando analisadas simultaneamente com as variáveis dos demais fatores abordados neste estudo.

Da Tabela 2, nota-se que a variável Renda Nacional Bruta Per Capita é o elemento de maior importância nos Índices de Percepção da Corrupção, quando analisado de forma simultânea com as demais variáveis do modelo, possuindo um poder de influência de 40,6\%. Logo, infere-se que a Renda Nacional Bruta Per Capita, dentre as demais variáveis, é a melhor opção na formulação de políticas públicas para os países, quando se trata de reduzir o índice de percepção da corrupção.

Com relação à Hipótese 2 (as variáveis do Fator Social estão inversamente relacionadas à percepção da corrupção), os resultados não permitem a rejeição da mesma, visto que ambas variáveis, Renda Nacional Bruta Per Capita e Educação, ao serem analisadas simultaneamente com as variáveis dos demais fatores, além de serem significantes no modelo de regressão, conforme se intensificam, atuam reduzindo a corrupção. Este achado concorda com os Chong e Gradstein (2007), Apergis, Dincer e Payne (2010) e Mendonça e Fonseca (2012) ao proporem que a renda per capita elevada contribui para com a redução da corrupção. Em outras palavras, isso confirma que a pobreza faz com as pessoas com menor renda sejam mais suscetíveis a se corromperem, por não terem os meios necessários para sua subsistência.

Esse resultado contraria Dobson e Ramlogan-Dobson (2010), de que uma elevada renda per capita está associada a um aumento da corrupção na América Latina, pois nos países dessa região os referidos autores verificaram haver grande presença do setor informal. Campanha e Barboza (2019) mostram que isso acontece porque as pessoas recorrem à informalidade, devido ao aumento de custos causados pelas exigências para registro, taxas de licenciamento, impostos altos, ou trabalho rigoroso e regulamentos ambientais. Assim, os informais podem buscar corromper agentes públicos quando fiscalizados, com vistas a evitar sanções. Por isso, de forma mais ampla, na América Latina pode estar acontecendo o mesmo que Campanha e Barboza (2019, p. 49-48) mostraram sobre o Brasil, o desenvolvimento de políticas públicas para o apoio às empresas, "mediante criação de instituições, marco legal e normas tributárias específicas e a mobilização de mecanismos no âmbito das instituições públicas de suporte”.

Os achados também corroboram com Sofwan et al. (2018), Ferraz, Finan e Moreira (2012) e Asongu e Nwachukwu (2015) ao proporem que a educação é um dos elementos fundamentais para redução da corrupção na sociedade, pois uma educação eficiente auxilia na formação de uma mentalidade contrária à corrupção, conduzindo, assim, a sociedade no desenvolvimento de um caráter mais íntegro nas diversas situações do cotidiano. Cabe destacar que, no Brasil, assim como em muitos países, a escolaridade dos trabalhadores da construção civil é relativamente baixa (Valinote et al, 2014), o que pode, também, contribuir para o aumento da corrupção.

No que diz respeito à Hipótese 3 (as variáveis do Fator Jurídico estão inversamente relacionadas à percepção da corrupção), os resultados sugerem sua rejeição parcial, uma vez que as variáveis Independência Judicial e Estado de Direito, ao 
Research, Society and Development, v. 10, n. 9, e32010918207, 2021

(CC BY 4.0) | ISSN 2525-3409 | DOI: http://dx.doi.org/10.33448/rsd-v10i9.18207

serem analisadas de forma síncrona com as variáveis dos demais fatores, não foram estatisticamente significantes na percepção da corrupção a partir dos dados utilizados na construção do modelo de regressão.

O produto da análise simultânea das variáveis Integridade do Sistema Jurídico e Imparcialidade de Júri com as variáveis dos demais fatores, são significativas e inversamente relacionadas à corrupção, concordando com os achados de Uslaner (2008), Montes e Luna (2020) ao inferir que ambas contribuem para a redução da corrupção. Mas há um avanço em relação ao estudo de Bennedsen, Feldmann e Lassen (2011), pois eles mostram que a regressão bivariada pode ser afetada por problemas de endogeneidade, o que é mais difícil de acontecer com a regressão multivariada. As questões jurídicas podem ser explicadas pelo fato de que um sistema jurídico íntegro e imparcial garante que a corrupção não vale à pena, pois não ficará impune. Para o Brasil, esse resultado vai ao encontro das conclusões de Valarini e Pohlmann (2019), que identificaram um modus operandi de formação de um cartel, realizada por executivos seniores em benefício de empresas de construção civil. Disso resultou a criação de uma Lei Criminal Corporativa, de aplicação mais rígida, bem como penas mais pesadas e o encarceramento de executivos seniores, que mudou todo o sistema de governança brasileiro na luta contra o crime corporativo e a corrupção.

Por fim, acerca da Hipótese 4 (a variável do Fator Político está inversamente relacionada à percepção da corrupção), os achados propõem sua não rejeição, corroborando assim com Pellegrini (2011) e Saha et al. (2014), ao inferirem que a Liberdade Civil e Direitos Políticos contribuem para a redução da corrupção na sociedade. Além de concordar, parcialmente, com Jetter, Agudelo e Hassan (2015), ao concluírem que a redução da corrupção advinda da Liberdade Civil e Direitos Políticos tende a ocorrer somente em países mais ricos. No Brasil, Valarini e Pohlmann (2019) verificaram que a criação de leis que alteraram as regras para o financiamento de campanhas eleitorais, com a proibição de doações de empresas, fez com que a competição entre os partidos políticos tornasse a participação da sociedade civil mais equilibrada.

\section{Considerações Finais}

A pesquisa aqui discorrida, apresentou, portanto, como a percepção da corrupção dos países é influenciada pelas variáveis relacionadas aos fatores social, jurídico, político e econômico, quando analisadas de forma conjunta e simultânea. Desta forma, este estudo difere dos outros indicados no embasamento teórico, por meio das variáveis analisadas (conforme Tabela 1) ou pelo método estatístico aplicado (regressão linear múltipla).

Assim como foi visto, os resultados encontrados mostraram que as variáveis: Renda Nacional Bruta Per Capita, a Liberdade Civil e os Direitos Políticos, a Educação, a Integridade do Sistema Jurídico e a Imparcialidade do Júri, são estatisticamente significantes para explicar os Índices de Percepção da Corrupção dos países, quando analisadas simultaneamente.

Esperava-se que o PIB da Construção Civil fosse significante ao explicar os Índices de Percepção da Corrupção dos países, porém os resultados estatísticos não foram suficientes para considerá-la como tal, bem como os resultados encontrados para as variáveis Independência Judicial, Inflação, Estado de Direito e Crescimento do PIB, contradizendo assim os resultados de Lučić, Radišić e Dobromirov (2016), Cieślik e Goczek (2018), Akça, Ata e Karaca (2012), Cordis (2009), Mungiu-Pippidi e Dadašov (2016) e Elbahnasawy e Revier (2012). Este achado indica que, na busca pela redução da corrupção em uma sociedade, as referidas variáveis não são estatisticamente relevantes para tal efeito.

Os achados também confirmaram as inferências de Chong e Gradstein (2007), Apergis, Dincer e Payne (2010), Mendonça e Fonseca (2012), Sofwan et al. (2018), Ferraz, Finan e Moreira (2012), Asongu e Nwachukwu (2015), Uslaner (2008), Montes e Luna (2020), Bennedsen, Feldmann e Lassen (2011), Pellegrini (2011) e Saha et al. (2014), no sentido de que as variáveis Renda Nacional Bruta Per Capita, Liberdade Civil e Direitos Políticos, Educação, Integridade do Sistema Jurídico 
e Imparcialidade do Júri, são verdadeiramente significantes nos Índices de Percepção da Corrupção dos países e, neste estudo, por meio da atuação simultânea com as demais variáveis independentes.

Logo, os resultados estatísticos desta pesquisa mostraram elevada confiabilidade, em virtude do valor obtido para o coeficiente de determinação do modelo $\left(\mathrm{R}^{2}=0,907\right)$, desse modo sugere que a identificação das variáveis mais relevantes permite orientar os formuladores de políticas públicas dos países, para desenvolver programas e projetos que possam impactar na percepção da corrupção.

Conclui-se, portanto, que, em ordem e grau de importância, as variáveis mais relevantes para explicar os índices de percepção da corrupção são: Renda Nacional Bruta Per Capita (40,6\%), Liberdade Civil e Direitos Políticos (29,4\%), Imparcialidade do Júri (28,3\%), Integridade do Sistema Jurídico (13\%) e Educação (10,4\%), conforme visto nos coeficientes padronizados da Tabela 2.

Usando como variável de controle a Renda Nacional Bruta Per Capita média, considerada a mais importante, pois obteve o maior coeficiente padronizado, os países analisados foram divididos em dois grupos: o primeiro composto por 48 países com a maior renda per capita média e o segundo composto por 74 países com menor renda per capita média.

Fazendo a análise de regressão com os dois grupos, verificou-se que o modelo contendo os países de maior renda per capita, apresentaram quatro das cinco variáveis que compuseram o modelo global, não sendo confirmada apenas a variável Integridade do Sistema Jurídico (conforme Tabela 6). No modelo oriundo dos países de menor renda per capita, verificou-se que três variáveis foram mantidas, sendo estas Renda Nacional Per Capita, Liberdade Civil e Direitos Políticos e Integridade do Sistema Jurídico (conforme Tabela 7). A manutenção das variáveis nos dois modelos mostra o quanto o modelo global é robusto.

Tabela 6. Resumo do Modelo de Regressão para Países com Menor Renda Per Capita Média - Renda Nacional Bruta Per Capita como Variável de Controle.

\begin{tabular}{|c|c|c|c|c|}
\hline \multirow{6}{*}{$\mathbf{R}^{2}=\mathbf{0 , 7 0 2}$} & Variáveis do Modelo & $\begin{array}{l}\text { Coeficientes não } \\
\text { Padronizados }\end{array}$ & $\begin{array}{c}\text { Coeficientes } \\
\text { Padronizados }\end{array}$ & $\begin{array}{c}\text { Significância } \\
\text { Estatística }\end{array}$ \\
\hline & $\begin{array}{l}\text { Índice de Percepção da } \\
\text { Corrupção (constante) }\end{array}$ & 7,081 & & 0,004 \\
\hline & $\begin{array}{l}\text { Renda Nacional Bruta } \\
\text { Per Capita }\end{array}$ & 3,509 & 0,270 & 0,000 \\
\hline & $\begin{array}{l}\text { Liberdade Civil e } \\
\text { Direitos Políticos }\end{array}$ & 4,929 & 0,425 & 0,000 \\
\hline & Independência Judicial & 2,372 & 0,476 & 0,000 \\
\hline & $\begin{array}{l}\text { Integridade do Sistema } \\
\text { Jurídico }\end{array}$ & 0,843 & 0,168 & 0,019 \\
\hline
\end{tabular}

Fonte: Autores (2021). 
Tabela 7. Resumo do Modelo de Regressão para Países com Maior Renda Per Capita Média - Renda Nacional Bruta Per Capita como Variável de Controle

\begin{tabular}{|c|c|c|c|c|}
\hline \multirow{7}{*}{$R^{2}=0,890$} & Variáveis do Modelo & $\begin{array}{c}\text { Coeficientes não } \\
\text { Padronizados }\end{array}$ & $\begin{array}{l}\text { Coeficientes } \\
\text { Padronizados }\end{array}$ & $\begin{array}{c}\text { Significância } \\
\text { Estatística }\end{array}$ \\
\hline & $\begin{array}{l}\text { Índice de Percepção da } \\
\text { Corrupção (constante) }\end{array}$ & $-52,805$ & & 0,000 \\
\hline & $\begin{array}{l}\text { PIB da Construção } \\
\text { Civil }\end{array}$ & 1,452 & 0,150 & 0,012 \\
\hline & $\begin{array}{l}\text { Renda Nacional Bruta } \\
\text { Per Capita }\end{array}$ & 3,949 & 0,361 & 0,000 \\
\hline & $\begin{array}{l}\text { Liberdade Civil e } \\
\text { Direitos Políticos }\end{array}$ & 11,199 & 0,466 & 0,000 \\
\hline & Índices de Educação & 51,171 & 0,199 & 0,007 \\
\hline & Imparcialidade do Júri & 3,562 & 0,389 & 0,000 \\
\hline
\end{tabular}

Fonte: Autores (2021).

Dentre as possíveis limitações atribuídas a esta pesquisa, assinala-se o fato de que os resultados devem ser interpretados limitando-se aos dados de 125 países. Essa limitação dá-se em função da falta de dados sobre os demais países reconhecidos pela United Nations (2020). Além disso, não foram encontrados dados referentes a outras variáveis que pudessem influenciar na corrupção, conforme a taxonomia de Rose-Ackerman e Palifka (2016), descrita na Figura 1, o que levou a que não fossem incluídas no modelo inicial.

Não obstante, as evidências empíricas encontradas acrescentam novos argumentos ao se olhar a questão da corrupção nos países e, como sugestão de trabalhos futuros, recomenda-se analisar as variáveis estudadas em séries temporais, para verificar como seria o comportamento do índice de percepção da corrupção dos países ao longo do tempo, para assim determinar se a relação que foi encontrada nesta pesquisa se mantém ou se há mudanças de comportamento.

Como sugestão para trabalhos futuros, verificar por meio de uma série temporal histórica, num período compreendido de 5 anos ou mais, se os resultados encontrados nesta pesquisa permanecem, para o referido período analisado ou se há diferenças significativas.

\section{Referências}

Akça, H., Ata, A. Y., \& Karaca, C. (2012). Inflation and Corruption Relationship: Evidence from Panel Data in Developed and Developing Countries. International Journal of Economics and Financial Issues, 2(3), 281-295;

Alatas, S.H. (1990). Corruption: Its Nature, Causes and Functions. Waltham Abbey, HERTS, United Kingdom: Avebury;

Alves, A.F., Curta, L.C., \& Dias, L.S. (2017). Analysis of Correlation among HDI (Human Development Index), Violence and Corruption Perceptions Index. [Conference Paper] Conference: 5th CR3+ Conference - Making Corporate Responsibility UsefulAt: Helsinki, Finland. https://www.researchgate.net/publication/317281178.

American Society of Civil Engineers. (2004). Civil Engineers Call for Global Standards to Curb Trillion Dollar Worldwide Corruption. Reston: ASCE;

Anaman, K. A. \& Amponsah, C. (2007). Analysis of the Causality Links Between the Growth of the Construction Industry and the Growth of the Macro Economy in Ghana. Acra: Institute of Economic. https://doi.org/10.1080/01446190701411208;

Apergis, N., Dincer, O. C., \& Payne, J. E. (2010). The relationship between corruption and income inequality in U.S. states: evidence from a panel cointegration and error correction model. Public Choice, 145(1-2), 125-135. https://doi.org/10.1007/s11127-009-9557-1;

Ashforth, B. E., Gioia, D. A., Robinson, S. L., \& Treviño, L. K. (2008). Re-Viewing Organizational Corruption. Academy of Management Review, 33(3), 670684. https://doi.org/10.5465/amr.2008.32465714; 
Research, Society and Development, v. 10, n. 9, e32010918207, 2021

(CC BY 4.0) | ISSN 2525-3409 | DOI: http://dx.doi.org/10.33448/rsd-v10i9.18207

Asongu, S., \& Nwachukwu, J.C. (2015). The Incremental Effect of Education on Corruption: Evidence of Synergy from Lifelong Learning. Economics Bulletin, 35(4), 2288-2308. 10.2139/ssrn.2661448;

Bennedsen, M., Feldmann, S. E., \& Lassen, D. D. (2011). Lobbying and Bribes - A Survey-Based Analysis of the Demand for Influence and Corruption. CESifo Working Paper Series 3496, CESifo;

Boyd, J. M., \& Padilha, J. D. (2009). FIDIC and Integrity: A Status Report. Leadership and Management in Engineering, 9(3), 125-128. https://doi.org/10.1061/(ASCE)1532-6748(2009)9:3(125);

Brol, M. (2016). Institutional determinants of corruption. Ekonomia i Prawo. Economics and Law, 15(1), 21-32. http://dx.doi.org/10.12775/eip.2016.002;

Campanha, L. J. \& Barboza, R. A. B. Quadro Jurídico de apoio aos Pequenos Empreendimentos: Lei do MEI, limitações e potencialidades. Revista Brasileira de Direito Empresarial, 5(2), p. 37 - 57.

Chan, A. P., \& Owusu, E. K. (2017). Corruption forms in the construction industry: Literature review. Journal of Construction Engineering and Management, 143(8), 040170571. https://doi.org/10.1061/(ASCE)CO.1943-7862.0001353;

Chong, A. \& Gradstein, M. (2007) Inequality and institutions. The Review of Economics and Statistics, 89, 454-465. https://doi.org/10.1162/rest.89.3.454;

Cieślik, A., \& Goczek, Ł. (2018). Control of corruption, international investment, and economic growth - Evidence from panel data. World Development, 103(March), 323-335. 10.1016/j.worlddev.2017.10.028;

Cordis, A. S. (2009). Judicial Checks on Corruption in the United States (February 1, 2008). Economics of Governance, 10(4), 375-401. https://ssrn.com/abstract=1019897;

Dobson, S., \& Ramlogan-Dobson, C. (2010). Is there a trade-off between income inequality and corruption? Evidence from Latin America. Economics Letters, 107(2), 102-104. 10.1016/j.econlet.2009.12.038;

Elbahnasawy, N. G., \& Revier, C. F. (2012). The determinants of corruption: Cross-country-panel-data analysis. The Developing Economies, 50(4), 311-333. https://doi.org/10.1111/j.1746-1049.2012.00177.x;

Ferraz, C., Finan, F., \& Moreira, D. B. (2012). Corrupting learning: Evidence from missing federal education funds in Brazil. Journal of Public Economics, 96(9-10), 712-726. 10.1016/j.jpubeco.2012.05.012;

Fonseca, J. J. (2002). Metodologia da Pesquisa Científica. UEC;

Gil, A. C. (2008). Métodos e técnicas de pesquisa social. Atlas;

Goel, R. K., \& Nelson, M. A. (2010). Causes of corruption: History, geography and government. Journal of Policy Modeling, 32(4), 433-447. https://doi.org/10.1016/j.jpolmod.2010.05.004;

Hair Jr., J.F., Black, W.C., Babin, B.J., \& Anderson, R.E. (2019). Multivariate data analysis. [9th Edition] Andover: Cengage Learning.

Ive, G., \& Gruneberg, L. (2000). The Economics of the Modern Construction Sector. United Kingdom: Palgrave Macmillan.;

Jetter, M., Agudelo, A. M., \& Hassan, A. R. (2015). The effect of democracy on corruption: Income is key. World Development, 74(C), 286-304. 10.1016/j.worlddev.2015.05.016;

Journal of Management Inquiry (2017). Special Issue: Corruption Part $1 \&$ Special Issue: Corruption Part 2. JMI, 26(3 \& 4), 247- 442

Kline, R. B. (2010). Principles and Practice of Structural Equation Modeling. The Guilford Press;

Kolstad, I., \& Wiig, A. (2016). Does democracy reduce corruption? Democratization, 23(7), 1198-1215. https://doi.org/10.1080/13510347.2015.1071797;

Luciano, E. M., Wiedenhöft, G. C., \& Callegaro, S. (2019). Entendendo os fatores de combate à corrupção: proposta de um constructo de dissuasão à corrupção. Revista de Contabilidade e Organizações, n. temático Fraudes e Corrupção: o que Contabilidade e Organizações têm a dizer? 20-30. http://dx.doi.org/10.11606/issn.1982-6486.rco.2019.158516;

Lučić, D., Radišić, M., \& Dobromirov, D. (2016). Causality between corruption and the level of GDP, Economic Research-Ekonomska Istraživanja, 29(1), 360-379, 10.1080/1331677X.2016.1169701;

Martins, G. A. \& Theóphilo, C. R. (2016). Metodologia da investigação científica para ciências sociais aplicadas. Atlas;

Mahalanobis, P C (1936). On the generalised distância in statistics. Proceedings of the National Institute of Sciences of India. 2(1), 49-55. http://bayes.acs.unt.edu:8083/BayesContent/class/Jon/MiscDocs/1936_Mahalanobis.pdf

Medeiros, C.R.O, \& Silveira, R.A. (2017). A Petrobrás nas teias da corrupção: mecanismos discursivos da mídia brasileira na cobertura da Operação Lava Jato. Revista de Contabilidade e Organizações, 31, 11-20. http://dx.doi.org/10.11606/rco.v11i31.134817;

Mendonça, H. F., \& Fonseca, A. O. (2012). Corruption, income, and rule of law: empirical evidence from developing and developed economies. Brazilian Journal of Political Economy, 32(2), 305-314. https://doi.org/10.1590/S0101-31572012000200009;

Montes, G. C., \& Luna, P. H. (2020). Fiscal transparency, legal system and perception of the control on corruption: empirical evidence from panel data. Empirical Economics, 1-33. 10.1007/s00181-020-01849-9; 
Research, Society and Development, v. 10, n. 9, e32010918207, 2021

(CC BY 4.0) | ISSN 2525-3409 | DOI: http://dx.doi.org/10.33448/rsd-v10i9.18207

Mungiu-Pippidi, A., \& Dadašov, R. (2016). Measuring control of corruption by a new index of public integrity. European Journal on Criminal Policy and Research, 22(3), 415-438. https://doi.org/10.1007/s10610-016-9324-z;

Nordin, R. M., Takim, R., \& Nawawi, A. H. (2013). Behavioural Factors of Corruption in the Construction Industry. Procedia - Social and Behavioral Sciences, 105, 64-74. 10.1016/j.sbspro.2013.11.008;

Nye, J. S. (1967). Corruption and Political Development: A Cost-Benefit Analysis. American Political Science Review, 61(2), 417-427. https://doi.org/10.2307/1953254;

Oberoi, R. (2014). Mapping the Matrix of Corruption: Tracking the Empirical Evidences and Tailoring Responses. Journal of Asian and African Studies, 49(2), 187-214. https://doi.org/10.1177/0021909613479319;

O'Connor, S., \& Fischer, R. (2012). Predicting Societal Corruption Across Time: Values, Wealth, or Institutions? Journal of Cross-Cultural Psychology, 43(4), 644-659. https://doi.org/10.1177/0022022111402344;

Pellegrini, L. (2011). Causes of corruption: a survey of cross-country analyses and extended results. In: Lorenzo Pellegrini, Corruption, development and the environment. [Chapter 3], pp. 29-51, Dordrecht: Springer Netherlands;

Revista de Contabilidade e Organizações (2019). Fraudes e Corrupção: o que Contabilidade e Organizações têm a dizer? RCO, n. temático, 1-85.

Rose-Ackerman, S. \& Palifka, B. J. (2016). Corruption and government: Causes, consequences, and reform. Cambridge University Press;

Saha, S., Gounder, R., Campbell, N., \& Su, J.J. (2014). Democracy and corruption: A complex relationship. Crime, Law and Social Change, 61(3), 287-308. DOI 10.1007/s10611-013-9506-2;

Sofwan, M., Setiono, P., Mohamed Elsayed, A. E., \& Saudagar, F. (2018). Fighting against Corruption through Character Education: The Voices of Indonesian Primary School Teachers. Indonesian Research Journal in Education, 2(2), 86-96. https://doi.org/10.22437/irje.v2i2.6092;

Stansbury, N. (2005). Exposing the foundations of corruption in construction. In y Diana Rodriguez, Gerard Waite and Toby Wolfe, Global Corruption Report 2005, pp 36-50. Pluto Press;

Tabachnick, B. G., \& Fidell, S. L. (1996). Using multivariate statistics. HarperCollins College Publishers;

Transparency International (2002). Bribe payer index 2002. https://www.transparency.org/research/bpi.

Transparency International (2018). What is corruption? https://www.transparency.org/what-is-corruption

Transparency International (2019). Bribe payer index 2019. https://www.transparency.org/research/bpi.

Unctad. (2000). Regulation and Liberalization in the Construction Services Sector and Its Contribution to the Development of Developing Countries. New York: UNCTAD;

United Nations. (2020). List of UN Member States. https://www.un.org/en/member-states/index.html.

Uslaner, E. (2008). Corruption, Inequality, and the Rule of Law: The Bulging Pocket Makes the Easy Life. Cambridge: Cambridge University Press. 10.1017/CBO9780511510410.003;

Uyanik, G. K., \& Guler, N. (2013). A Study on Multiple Linear Regression Analysis. Procedia - Social and Behavioral Sciences, 106(10), 234-240. https://doi.org/10.1016/j.sbspro.2013.12.027;

Valinote et al. (2014). Análise da Qualidade de Vida, Capacidade para o Trabalho e Nível de Estresse em Trabalhadores da Construção Civil. Revista Brasileira de Ciências Ambientais Vol 32, 115-126.

Valarini, E. \& Pohlmann, M. (2019). Organizational Crime and Corruption in Brazil: a Case Study of the Operation Carwash Court Records. International Journal of Law, Crime and Justice, Vol. 59, 1-15.

Williams, M. N., Grajales, C. A. G., \& Kurkiewicz, D. (2013). Assumptions of Multiple Regression: Correcting Two Misconceptions. Practical Assessment, Research, and Evaluation, 18(11). https://doi.org/10.7275/55hn-wk47;

Zyglidopoulos, S., Hirsch, P., Holan, P. M., \& Phillips, N. (2017). Expanding Research on Corporate Corruption, Management, and Organizations. Journal of Management Inquiry, 26(3), 247-253. 10.1177/1056492617706648. 